
\title{
$\angle S$ Research Square \\ Comparison of Retinal Vascular Arcade Trajectory Between Eyes with an Idiopathic Macular Hole and the Healthy Fellow Eye
}

\section{Hossein Nowroozzadeh}

Shiraz University of Medical Sciences

Shadi Meshksar ( $\nabla$ shad.moshksar.73@gmail.com )

Shiraz University of Medical Sciences

Ali Azimi

Shiraz University of Medical Sciences

Ali Rasti

Shiraz University of Medical Sciences

Ahad Sedaghat

Iran University of Medical Sciences

\section{Research Article}

Keywords: Macular hole, Retinal vessel trajectory, Traction.

Posted Date: September 27th, 2021

DOI: https://doi.org/10.21203/rs.3.rs-327248/v1

License: (c) (i) This work is licensed under a Creative Commons Attribution 4.0 International License. Read Full License

Version of Record: A version of this preprint was published at International Ophthalmology on January 18th, 2022. See the published version at https://doi.org/10.1007/s10792-022-02221-9. 


\section{Abstract}

Purpose: We aimed to present a method to evaluate the retinal arcade vascular trajectory by measuring the distance between the retinal veins in eyes with idiopathic macular hole (IMH) and compare this measurement with healthy fellow eyes.

Methods: In this Cross-sectional study 18 patients with unilateral IMH were enrolled. We used standard fundus photographs, which were obtained from each eye with a digital fundus camera using a 55-degree lens. The calculation of the retinal arcade vascular trajectories was done by drawing and measuring 5 vertical lines within the macular area.

Results: The mean age of the patients was $65 \pm 9$ years. The between-groups differences of each vertical line were not statistically significant, except for the differences between the most temporal line, which was greater in the diseased group (9388 vs. $8322 \mathrm{~mm} ; \mathrm{P}=0.034$ ). The ratio between the $5^{\text {th }}$ (most temporal) and the third (fovea-center) vertical lines was greater than 1 (V-shape) in $72 \%$ of eyes with a macular hole, whereas it was less than one (U-shape) in $78 \%$ of control eyes $(P=0.003)$.

Conclusions: We demonstrated that in eyes with an IMH the vascular arcade has more tendency to diverge on its path temporal to the fovea.

\section{Introduction}

Macular hole is a full-thickness defect of the retinal tissue involving the anatomic fovea, and primarily, the foveola; thereby causing significant visual loss and metamorphopsia. [1] Macular holes could be found in highly myopic eyes or following ocular trauma, but the great majority are idiopathic. [1, 2] Idiopathic Macular Hole (IMH), with the approximate prevalence of 3.3 per 1,000 people, [3] usually affects healthy individuals with normal refractive errors in their sixth or seventh decades of life, and roughly two-thirds of the involved patients are females. [3,4] The risk of fellow eye involvement has been reported as $15.6 \%$ (range 3 to $22 \%$ ). [5]

The primary cause of IMH is supposed to be originated from the abnormal attachment of the posterior hyaloid to the fovea during the process of posterior vitreous detachment, which exerts adequate force to detach and tear off the central fovea that eventually evolves into a full-thickness macular hole. $[1,2,6]$ Nonetheless, the macular hole could be spontaneously closed, provided that the posterior vitreous has detached completely from the macula. [1, 2] However, the tangential forces from the surrounding retina might prevent a fresh macular hole from successful closure. A typical vitrectomy for IMH usually includes induction of posterior vitreous detachment (if not already occurred) plus peeling of the internal limiting membrane of the retina for about 1- to 2-disc diameters. [7-9] The latter procedure would relax the retina and decrease the mentioned tangential forces to maximize the chance of macular hole closure.

Therefore, the tangential forces across the retina may have a role in the development of an IMH or its persistence after surgery. It is difficult to measure the retinal tangential force in vivo. The retinal vessels 
pass through the inner retina, and hence the trajectory of the arcade vessels may be correlated with retinal tangential forces at the macula. In this study, we aimed to present a method to evaluate the trajectory of arcade vessels and study its association with the development of IMH.

\section{Materials And Methods}

\section{Subjects}

In this cross-sectional study, patients with unilateral IMH who referred to Khalili hospital, Shiraz, Iran, from February 2017 to March 2019 were included. The diagnosis of IMH was confirmed by a retinal specialist (MHN) according to physical examination and OCT findings. We excluded eyes with concomitant glaucoma, myopic macular hole, diabetic retinopathy, retinal dystrophies, or previous ocular trauma, retinal detachment, or any serious ophthalmic problem. The normal fellow eyes should also have no macular hole of any stage, vitreomacular traction or epiretinal membrane. Finally, 18 patients were enrolled in the study.

Informed consent was obtained from all participants. The protocol of the study was approved by the Ethics Committee at Shiraz University of Medical Sciences and adhered to the principles of the Declaration of Helsinki.

\section{Measurements}

For assessing the trajectory of arcade vessels, we used fundus photographs which were obtained with an OCT device equipped with a digital fundus camera (Heidelberg Engineering, Heidelberg, Germany) using a 55-degree lens to create a wider field of the image. A single expert examiner took all images with the same camera. Then, the images were transferred to the Photoshop CS6 interface for measurements. The calculation of the arcade vessels' trajectories was performed by measuring the distance between the upper and the lower temporal retinal veins in 5 vertical lines. The first distance (D1) crossed the temporal border of the optic disc. The second line (D2) crossed the median distance from the center of the fovea to the temporal border of the optic disc. The third line (D3) crossed the center of the fovea. The fourth and fifth distances (D4 \& 5) were mirror images of the second and the first lines according to the center of the macula, respectively. We also measured the distance between the fovea and the disc, and also the distances of the fovea from the superior and the inferior veins through the third vertical line (as described previously) (Fig. 1). The logic behind picking the veins (instead of arteries) for measurements was our initial observation of all images, in which the arcade veins showed less variability in branching than arteries. After each branching of the temporal veins, we chose the thicker vein as the main branch for measurement. To mask the IMH eyes from the examiner who measured the mentioned lines, the fovea of all fundus images was covered with a digital square shield prior to the analyses.

\section{Statistical analysis}


All analyses were performed using the SPSS software version 21 . The normality of the data was assessed by the Kolmogorov-Smirnov test. Paired t-test or Wilcoxon signed-rank test were used to compare the variables between fellow eyes (IMH vs. normal), when the data were distributed normally or not, respectively. A P value of less than 0.05 was considered significant.

\section{Results}

Overall, data from both eyes of 18 patients with unilateral IMH were gathered and analyzed. The baseline characteristics of the patients and their eyes are summarized in Table 1.

Table 1

Baseline characteristics of the participants and their eyes (macular hole vs. the fellow control eyes).

\begin{tabular}{|c|c|c|c|c|}
\hline & All & Diseased Eye & Fellow eye & P Value \\
\hline Age, y & $65 \pm 9$ & & & \\
\hline Sex, (M/F) & $8 / 10$ & & & \\
\hline Laterality, (R/L) & & $10 / 8$ & $8 / 10$ & 0.505 \\
\hline Axial Length, mm & & $23.0 \pm 0.6$ & $22.9 \pm 0.7$ & 0.079 \\
\hline
\end{tabular}

There was no significant difference in fovea-disc, fovea-superior vein, fovea-inferior vein, and D1-4 distances between the diseased and control eyes (Table 2). The D5 was significantly greater in IMH eyes than healthy eyes $(9388 \pm 1794$ vs. $8322 \pm 1525 \mathrm{~mm} ; \mathrm{P}=0.034)$. 
Table 2

Comparison of macular geometric parameters between the eyes with macular hole and the fellow control eyes (based on the measurements on Photoshop CS6 interface).

\begin{tabular}{|lccl|}
\hline & Diseased Eye & Fellow eye & P Value \\
\hline Fovea-Disc distance, $\mu \mathrm{m}$ & $4506 \pm 422$ & $4645 \pm 400$ & 0.173 \\
\hline Fovea-SV distance, $\mu \mathrm{m}$ & $4422 \pm 742$ & $5003 \pm 1281$ & 0.093 \\
\hline Fovea-IV distance, $\mu \mathrm{m}$ & $4270 \pm 918$ & $4470 \pm 1148$ & 0.576 \\
\hline D1, $\mu \mathrm{m}$ & $4696 \pm 1480$ & $5277 \pm 1551$ & 0.228 \\
\hline D2, $\mu \mathrm{m}$ & $7522 \pm 1280$ & $8152 \pm 1375$ & 0.130 \\
\hline D3, $\mu \mathrm{m}$ & $8692 \pm 1370$ & $9473 \pm 1974$ & 0.104 \\
\hline D4, $\mu \mathrm{m}$ & $9189 \pm 1352$ & $9055 \pm 1584$ & 0.698 \\
\hline D5, $\mu \mathrm{m}$ & $9388 \pm 1794$ & $\mathbf{8 3 2 2 \pm 1 5 2 5}$ & $\mathbf{0 . 0 3 4}$ \\
\hline D1-5, refer to text and figure-1 for definition. & & \\
\hline IV, inferior arcade vein; SV, Superior arcade vein. & \\
\hline
\end{tabular}

The proportion of D3 to D1 showed no statistical difference between the groups, whereas the D5/D3 was significantly greater in the IMH group than controls $(1.09 \pm 0.18$ vs. $0.93 \pm 0.16$, respectively; $P=0.006)$ (Fig. 2).

We further categorized the eyes into V-shape arcade (D5/D3 $>1)$ and U-shape arcade $(D 5 / D 3=<1)$. Considering this definition, most of the eyes (72\%) with IMH had V-shape arcades, while $78 \%$ of the control eyes had U-shape arcades $(P=0.003$; Figs. 3 and 4$)$.

\section{Discussion}

This study showed that the arcade veins tended to adopt a more divergent path in eyes with IMH than the control eyes. This divergence in vascular paths was particularly evident temporal to the fovea. As a result, eyes with IMH might have a more chance to assume a V-shape arcade in contrast to the normal eyes which usually takes a U-shape path.

The results of our study are in line with Yoshihara et al. [10] who demonstrated that the eyes with IMH have a significantly wider retinal artery trajectory compared to the normal fellow eyes. Our study, however, has several differences from Yoshihara et al. in terms of the methods used to assess the arcade vessel trajectory. They picked retinal arteries, while we chose retinal veins. The logic behind choosing the veins over the arteries in our study was provided in the method section. Compared to Yoshihara et al., we also used a completely different and simpler way to measure retinal vascular paths. Moreover, their method mostly included the vascular path between the fovea and the disc, while we also analyzed the same 
distance temporal to the fovea. Anyway, with its distinct method, the present study confirmed the findings of Yoshihara et al. regarding a wider retinal vessel trajectory in eyes with $\mathrm{IMH}$.

The fovea of the human eye is subjected to two main tractional forces from the posterior surface of the vitreous and tangential forces from the surrounding retina. The vitreous traction is supposed to be the most important cause of developing IMHs. $[1,11]$ The fovea would be torn off if the mentioned forces overcome its tensile strength. For eyes with a persistent attachment of the vitreous to the edges of the macular hole, vitrectomy is mandatory to resolve the vertical (z-axis) traction from the vitreous, and this step is considered the most important phase of the operation in these eyes. [12,13]

It is not thoroughly understood that why some macular holes with released vitreous traction are spontaneously closed, while others would persist and ultimately need surgical intervention. The flow of liquid vitreous through the macular hole and tangential forces from the surrounding retina are the major culprits. [1, 2] A typical example of retinal tangential forces is the development of epiretinal membrane in the macular area. [14] A more subtle traction is introduced by the internal limiting membrane; hence, internal limiting membrane peeling is considered an essential part of modern macular hole surgery. [7-9]

It is difficult to measure retinal tangential forces in vivo. Intuitively, the wider path of arcade vessels might be correlated with a greater tangential force at the fovea. These forces should probably exert their effect via the y-axis (Fig. 1-B). In our study, we did not find any intergroup difference in the fovea-disc distance, which could be correlated with tangential forces in the x-axis. These justifications, however, need to be verified by future studies.

In addition, since the vitreous is usually attached firmly to retinal vessels, the larger distance between arcade vessels can generate stronger tractional forces (from the remaining vitreo-foveal attachment) on the fovea by inertia or momentary force during eye movements, leading to an increased chance for macular hole formation. [10]

We found no significant difference between eyes with IMH and normal fellow eyes in vertical arcade vein distances and proportions nasal to the fovea, while we showed a significant difference in D5 and D5/D3, indicating a wider vascular path for eyes with macular hole temporal to the fovea. This finding might emphasize the importance of vertical tractional forces that are exerted temporal to the fovea rather than the nasal tractions. Considering the temporal venous paths, and based on the D5/D3 measurements, we introduced a classification (i.e., V-shape vs. U-shape), which might be clinically useful in a rapid distinction between eyes with probably more y-axis tangential traction (V-shape; and though a greater risk of $\mathrm{MH}$ formation) versus those with less traction (U-shape).

This study is limited by its relatively small sample size. However, it seems acceptable from a statistical point of view, since we had matched controls (fellow eyes). Involving the fellow eye as control could be a power of the present study because it removes most of the confounding factors such as age, sex, and also most ocular biometric features, which are typically similar in fellow eyes. However, since the fellow eyes of IMHs are at greater risk of developing macular holes, [15] the findings of this study should be 
confirmed by future studies with larger sample sizes that include normal patients as well. Finally, we used Photoshop CS6 interface to calculate retinal distances; and therefore, the measured distances (in micrometers) are not exactly the actual values (and are somehow magnified). However, this issue affected all images in the same manner, and so it did not compromise the validity of statistical analyses or calculated proportions.

\section{Conclusion}

Our study showed that the arcade veins adopt a more divergent path (particularly temporal to the fovea) in eyes with IMH than their fellow eyes. From a clinical point of view, a V-shape arcade might be associated with an increased risk of macular hole formation compared with a U-shape vascular path. This association, however, does not prove any cause-and-effect relationship; and such assumptions should be evaluated with appropriate prospective studies.

\section{Declarations}

Acknowledgment: The authors appreciate Dr. Yasaman Sadeghi for cooperation in data gathering.

Funding: No funds, grants, or other support was received.

Conflicts of interest/Competing interests: All authors declare that they do not have any conflict of interest.

Availability of data and material: Data will be available from the corresponding author on reasonable request.

Code availability: Not applicable.

Authors' contributions: Conception: MHN, ShM; design: MHN, ShM, AS; Data acquisition: AA, AR; data analysis: MHN, ShM; data interpretation: AA, AR, AS; drafting: MHN, ShM, AA, AR, AS Final approval: MHN, ShM, AA, AR, AS.

Ethics approval: This study was conducted in accordance to the tenets of the Declaration of Helsinki and its protocol was approved by the ethics committee at Shiraz university of Medical Sciences (Approval ID: IR.SUMS.MED.REC.1398.167)

Consent to participate: Informed consent was obtained from all participants.

Consent for publication: Not applicable.

\section{References}

1. Oh H (2014) Idiopathic macular hole. Dev Ophthalmol 54150-158. https://doi.org/10.1159/000360461 
2. la Cour M, Friis J (2002) Macular holes: classification, epidemiology, natural history and treatment. Acta Ophthalmol Scand 80(6):579-587. https://doi.org/10.1034/j.1600-0420.2002.800605.x

3. Madi HA, Masri I, Steel DH (2016) Optimal management of idiopathic macular holes. Clin Ophthalmol 1097 - 116. https://doi.org/10.2147/OPTH.S96090

4. Azzolini C, Patelli F, Brancato R (2001) Correlation between optical coherence tomography data and biomicroscopic interpretation of idiopathic macular hole. Am J Ophthalmol 132(3):348-355. https://doi.org/10.1016/s0002-9394(01)01005-4

5. Ezra E, Wells JA, Gray RH et al (1998) Incidence of idiopathic full-thickness macular holes in fellow eyes: A 5-year prospective natural history study. Ophthalmology 105(2):353-359. https://doi.org/10.1016/S0161-6420(98)93562-X

6. Johnson MW, Van Newkirk MR, Meyer KA (2001) Perifoveal Vitreous Detachment Is the Primary Pathogenic Event in Idiopathic Macular Hole Formation. Arch Ophthalmol 119(2):215-222. https://doi.org/10-1001/pubs.Ophthalmol.-ISSN-0003-9950-119-2-ecs00023

7. Chatziralli IP, Theodossiadis PG, Steel DHW (2018) Internal limiting membrane peeling in macular hole surgery; why, when, and how? Retina 38(5):870-882. https://doi.org/10.1097/iae.0000000000001959

8. Morescalchi F, Costagliola C, Gambicorti E et al (2017) Controversies over the role of internal limiting membrane peeling during vitrectomy in macular hole surgery. Surv Ophthalmol 62(1):58-69. https://doi.org/10.1016/j.survophthal.2016.07.003

9. Rahimy E, McCannel CA (2016) Impact of internal limiting membrane peeling on macular hole reopening: a systematic review and meta-analysis. Retina 36(4):679-687. https://doi.org/10.1097/iae.0000000000000782

10. Yoshihara N, Sakamoto T, Yamashita T et al (2015) Wider Retinal Artery Trajectories in Eyes with Macular Hole Than in Fellow Eyes of Patients with Unilateral Idiopathic Macular Hole. PLOS ONE 10(4):e0122876. https://doi.org/10.1371/journal.pone.0122876

11. Steel DH, Lotery AJ (2013) Idiopathic vitreomacular traction and macular hole: a comprehensive review of pathophysiology, diagnosis, and treatment. Eye (Lond) 27(Suppl 1):S1-S21. https://doi.org/10.1038/eye.2013.212 Suppl 1 ) .

12. Yee P, Sevgi DD, Abraham J et al (2020) iOCT-assisted macular hole surgery: outcomes and utility from the DISCOVER study. Br J Ophthalmol bjophthalmol-2020-316045. https://doi.org/10.1136/bjophthalmol-2020-316045

13. Mahmoud TH (2020) Insight into Long-Term Outcomes after Macular Hole Surgery. Ophthalmol Retina 4(4):377. https://doi.org/10.1016/j.oret.2019.11.012

14. Guber J, Scholl HPN, Valmaggia C (2019) Surgical Outcome after Lamellar Macular Hole Associated with Epiretinal Membrane. Ophthalmologica 241(1):56-60. https://doi.org/10.1159/000493349

15. Kumagai K, Ogino N, Hangai M, Larson E (2012) Percentage of Fellow Eyes That Develop FullThickness Macular Hole in Patients With Unilateral Macular Hole. Arch Ophthalmol 130(3):393-394. https://doi.org/10.1001/archopthalmol.2011.1427 


\section{Figures}
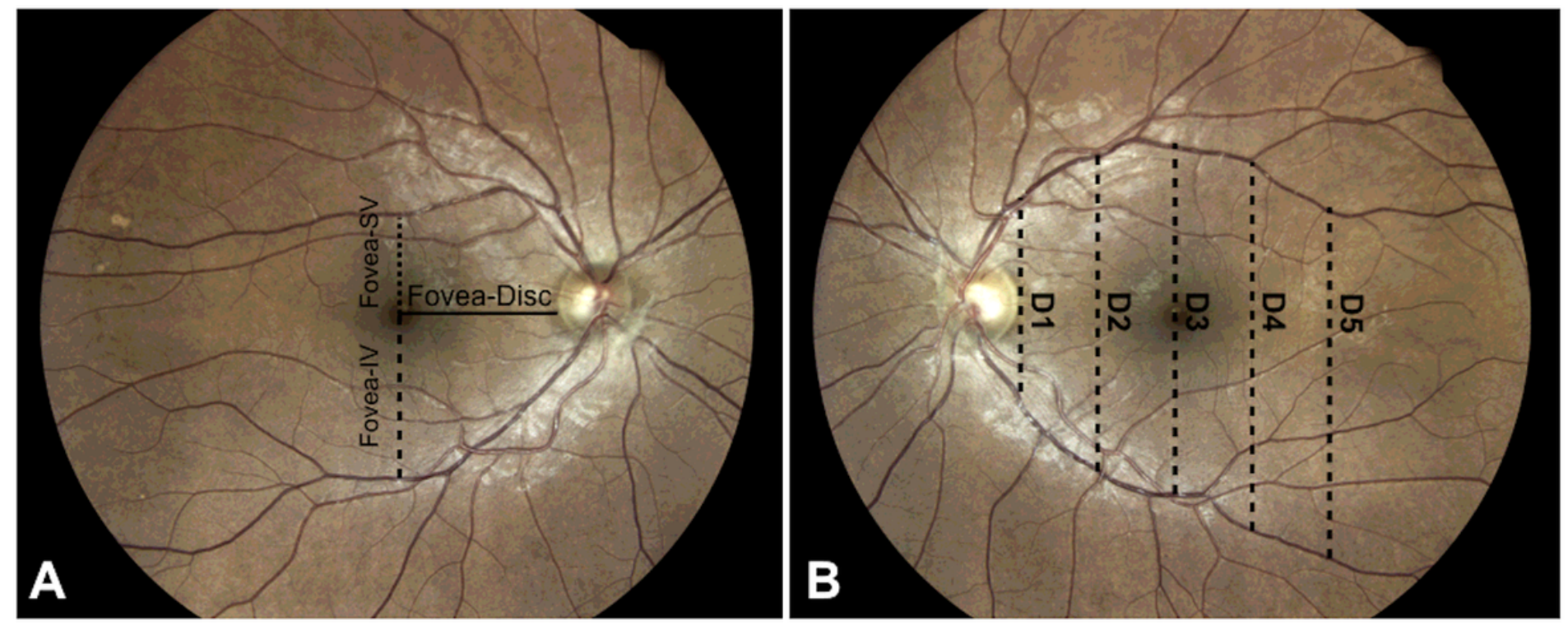

\section{Figure 1}

The method of the measurement of arcade vessels trajectory. A) Distance between the fovea and the disc; and distances between the fovea and superior (SV) and inferior (IV) arcade veins. B) D1 to D5 demonstrates equally-distanced vertical lines from the temporal margin of the disc (D1) to the most temporal line (D-5). 


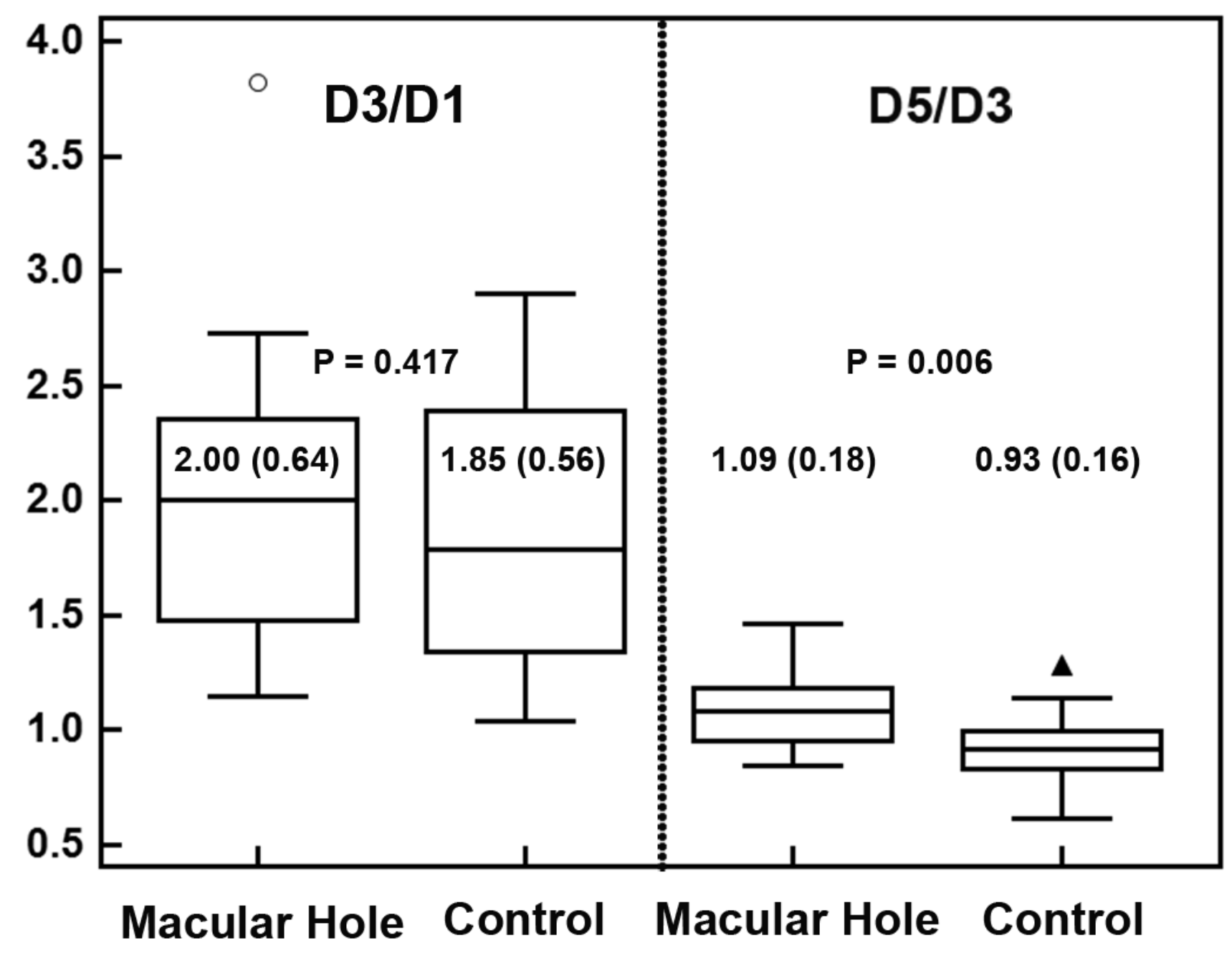

Figure 2

Box and whisker plots showing the comparison of D3/D1 and D5/D3 proportions between the eyes with idiopathic macular hole and control fellow eyes. 

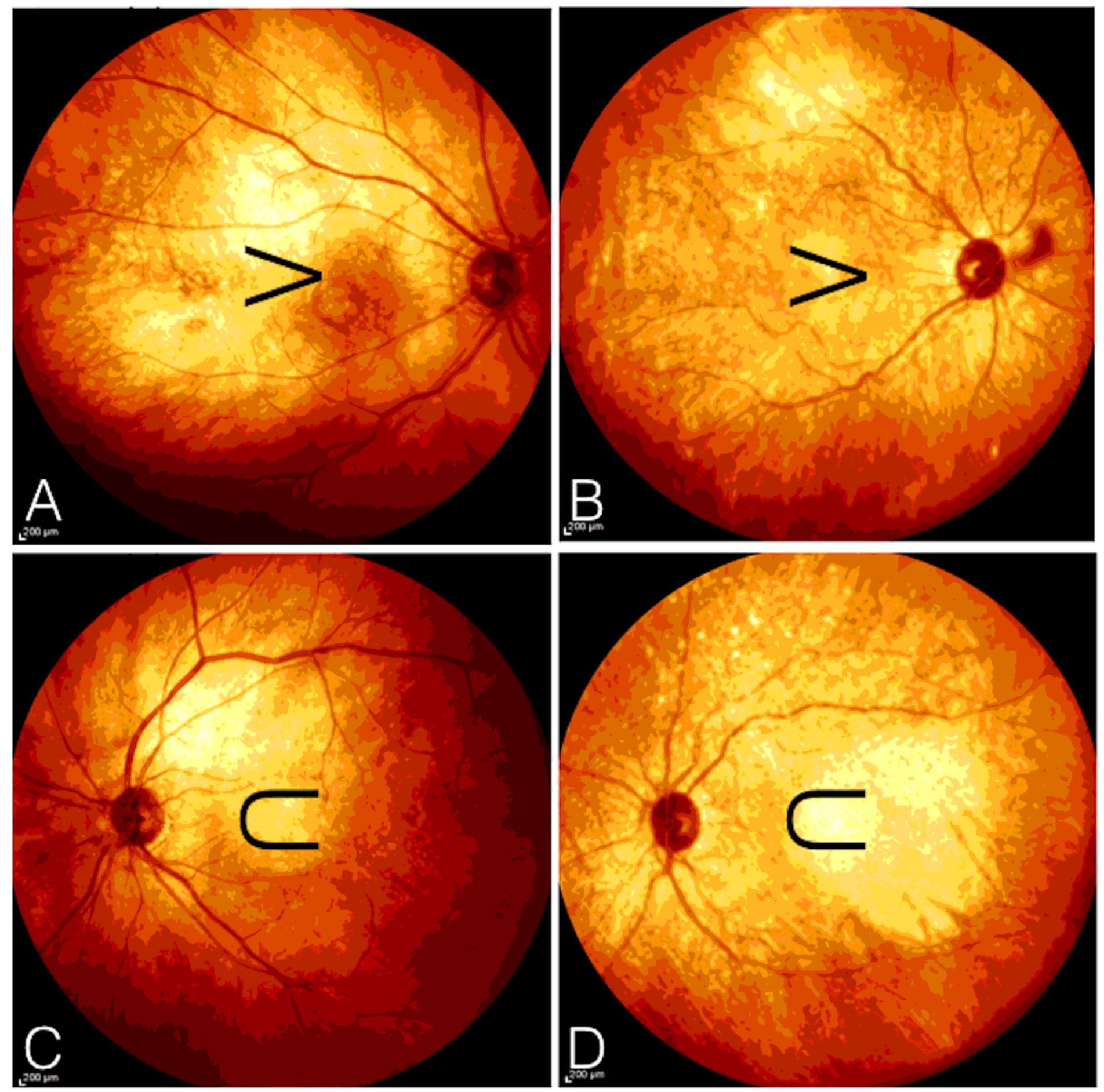

\section{Figure 3}

Typical V-shape vs. U-shape arcades in two cases with unilateral idiopathic macular hole. A \& B, the Vshape arcades in eyes with idiopathic macular hole. C \& D, the respective normal fellow eyes with the Ushape arcade vessels. 


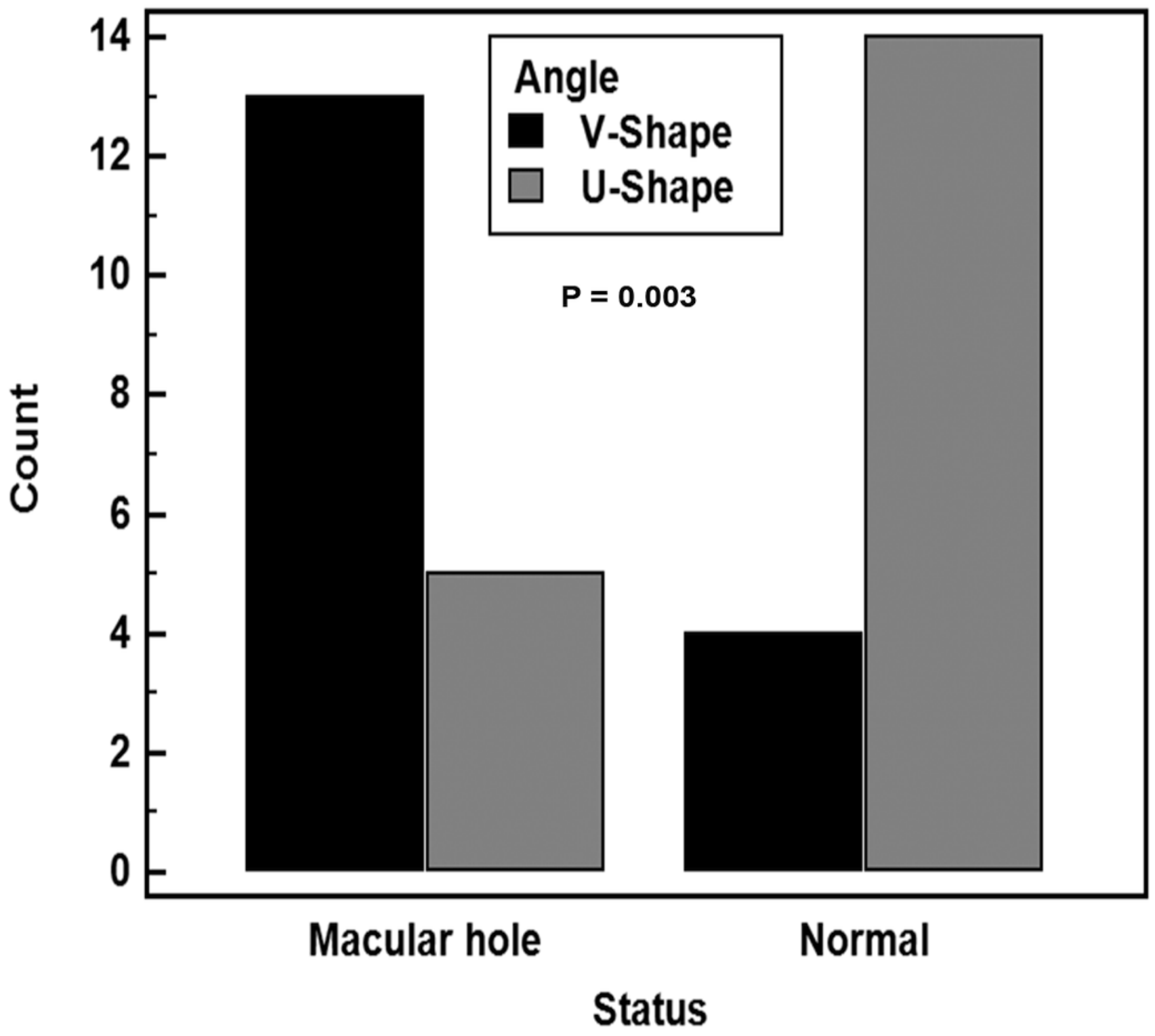

Figure 4

Bar chart demonstrating the frequency of V-shape vs. U-shape arcades in eyes with idiopathic macular hole vs. control fellow eyes. 